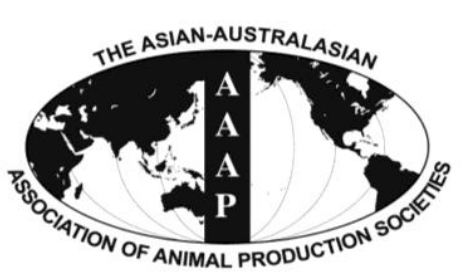

Open Access

Asian Australas. J. Anim. Sci.

Vol. 28, No. 1 : 14-19 January 2015

http://dx.doi.org/10.5713/ajas.14.0212

www.ajas.info

pISSN 1011-2367 elSSN 1976-5517

\title{
Genetic Diversity and Population Structure in Native Chicken Populations from Myanmar, Thailand and Laos by Using 102 Indels Markers
}

\author{
A. A. Maw ${ }^{1,2}$, K. Kawabe ${ }^{3, *}$, T. Shimogiri ${ }^{2}$, W. Rerkamnuaychoke ${ }^{4}$, Y. Kawamoto ${ }^{5}$, \\ S. Masuda ${ }^{6}$, and S. Okamoto ${ }^{2}$ \\ ${ }^{1}$ University of Veterinary Science, Yezin, Nay Pyi Taw 05282, Myanmar
}

\begin{abstract}
The genetic diversity of native chicken populations from Myanmar, Thailand, and Laos was examined by using 102 insertion and/or deletion (indels) markers. Most of the indels loci were polymorphic (71\% to 96\%), and the genetic variability was similar in all populations. The average observed heterozygosities $\left(H_{O}\right)$ and expected heterozygosities $\left(H_{E}\right)$ ranged from 0.205 to 0.263 and 0.239 to 0.381 , respectively. The coefficients of genetic differentiation (Gst) for all cumulated populations was 0.125 , and the Thai native chickens showed higher Gst (0.088) than Myanmar (0.041) and Laotian (0.024) populations. The pairwise Fst distances ranged from 0.144 to 0.308 among populations. A neighbor-joining (NJ) tree, using Nei's genetic distance, revealed that Thai and Laotian native chicken populations were genetically close, while Myanmar native chickens were distant from the others. The native chickens from these three countries were thought to be descended from three different origins $(K=3)$ from STRUCTURE analysis. Genetic admixture was observed in Thai and Laotian native chickens, while admixture was absent in Myanmar native chickens. (Key Words: Indels Markers, Genetic Admixture, Genetic Diversity, Native Chicken)
\end{abstract}

\section{INTRODUCTION}

Native chickens may be regarded as greatly diversified populations because of their long-term adaptation in response to varied agro-ecological zones. Native chickens possess unique adaptive traits that permit them to survive and reproduce under the harsh climatic, nutritional, and

\footnotetext{
* Corresponding Author: Kotaro Kawabe. Tel: +81-99-285-3591, Fax: +81-99-285-3582, E-mail: kawabe@gene4.agri.kagoshimau.ac.jp

${ }^{2}$ Faculty of Agriculture, Kagoshima University, Kagoshima 8900065, Japan.

${ }^{3}$ Natural Science Centre for Research and Education, Kagoshima University, Kagoshima 890-0065, Japan.

${ }^{4}$ Faculty of Veterinary Medicine, Kasetsart University, Bangkok 10900, Thailand.

${ }^{5}$ Faculty of Agriculture, University of Ryukyus, Nishihara 9030213, Japan.

${ }^{6}$ Liberal Arts Education Centre, Aso Campus, Tokai University, Minami Aso-mura 869-1404, Japan.

Submitted Mar. 24, 2014; Revised Jun. 5, 2014; Accepted Jul. 8, 2014
}

management conditions typically associated with low inputoutput production systems (Mwacharo et al., 2006). There are many kinds of native chickens in Asia. Most of them have not been improved and have lower productivity than the improved foreign breeds. Many kinds of native chickens can be found in all areas where farmers live. Farmers rear native chickens as important protein sources. Chickens were domesticated from the Red jungle fowl in Southeast Asia (Nishibori et al., 2005). Myanmar, Thailand, and Laos are three neighboring countries in Southeast Asia that share their borders on the Mekong River, which originates far away, in Quinghai Province, China. Native chickens have been domesticated in these countries for generations and have yielded a wide variety of chickens. It is very interesting to assess the genetic diversity of native chickens in those countries where native chickens are known to have been domesticated in the region of their ancestral species, Red jungle fowl.

Various tools offer a new approach to investigate both the genetic diversity and phylogenetic relationship among 
the sub-species of Gallus gallus and the domestic chicken. Some studies using blood protein variation in native chicken populations from Laos (Okamoto et al., 1999) and Myanmar (Okamoto et al., 2004) have been reported. Mitochondrial DNA sequencing is also a powerful tool for population genetic analysis. Liu et al. (2006) reported the geographical distribution of a wide range of domestic chickens in Eurasian regions. Kawabe et al. (2014) reported the genetic diversity of Laotian native fowls by mtDNA variation. The genetic characterization of Thai indigenous chickens and commercial chickens has been assessed by 20 microsatellite loci (Dorji et al., 2011). In addition, the genetic variation and phylogeographic analysis of native chicken populations from Myanmar and Thailand have been studied using 98 autosomal single nucleotide polymorphism (SNP) markers (Riztyan et al., 2012).

Insertion and/or deletion (indels) polymorphisms are diallelic markers with the potential for use in the study of genetic diversity and phylogenic relationships. Indels markers have many genetic advantages for analytical use, they are wide spread throughout the genome, all of the polymorphisms derive from a single mutation event, and they have reduced mutation rates (Natalle et al., 2010). In our previous study, we analyzed the genetic diversity of Myanmar and Indonesian native chickens together with two jungle fowl species using 102 indels polymorphisms (Maw et al., 2012). We found that genetic variability was higher among native chicken populations than in Red jungle fowl (Gallus gallus bankiva) and Green jungle fowl (Gallus varius). The native chickens from the two countries were genetically close to each other and distinct from Red and Green jungle fowl from Java Island, Indonesia.

In this study, we aimed to assess the genetic diversity and to clarify the population structure of native chicken populations from Myanmar, Thailand, and Laos by using 102 indels polymorphisms from previous studies as genetic markers.

\section{MATERIALS AND METHODS}

\section{Birds and DNA extraction}

A total of 278 blood samples were collected from nine native chicken populations, comprising three populations from each of three countries. The blood samples of Myanmar native chickens were collected from Yangon (YG) and Pegu (PG, Lower Myanmar) and from Mandalay (MD, Central Myanmar). From Thailand, the blood samples were collected from Trat and Chantaburi (TC, Eastern area), Kanchahaburi (KC, Western area), and from Nan, Chiang Rai and Lampang (NCL, Northern area). From Laos, the blood samples were collected from Viangchan and Louangphrabang (VC and LP, Central area) and from Pakxe (PK, Southern area). The sampled populations,
Table 1. Sampled populations, abbreviations and number of samples in each population

\begin{tabular}{llcc}
\hline Country & \multicolumn{1}{c}{ Population } & Abbreviation & $\begin{array}{c}\text { No. of } \\
\text { Sample }\end{array}$ \\
\hline Myanmar & Yangon & YG & 27 \\
& Mandalay & MD & 40 \\
& Pegu & PG & 13 \\
\multirow{5}{*}{ Thailand } & Trat and Chantaburi & TC & 15 \\
& Kanchahaburi & KC & 35 \\
& Nan, Chiang Rai, Lampang & NCL & 19 \\
Laos & Viangchan & VC & 50 \\
& Louangphrabang & LP & 44 \\
& Pakxe & PK & 35 \\
Total & & & 278 \\
\hline
\end{tabular}

abbreviations, and number of samples in each population are presented in Table 1. Genomic DNA was extracted from blood samples using standard phenol-chloroform extraction protocols (Sambrook, 1989). Detailed information and genotyping methods of the 102 indels markers used in this study ( $\mathrm{m} 1$ to $\mathrm{m} 102$ ) can be found in our previous study (Maw et al., 2012). The genotypes of the indels polymorphisms were determined by size differences among the PCR fragments.

\section{Statistical analysis}

The genetic variability of each population was assessed by calculating the proportion of polymorphic loci (Ppoly: Lewontin and Hubby, 1966), and average observed heterozygosity $\left(H_{O}\right)$ and expected heterozygosity $\left(H_{E}\right)(\mathrm{Nei}$, 1978). A chi square $\left(x^{2}\right)$ approximation was used to test for Hardy-Weinberg equilibrium (HWE; Weir, 1996) and calculate the degree of genetic differentiation (Gst; Nei, 1973). Using Fstat version 2.9.3.2, pair-wise distance matrix (Fst) values (Weir and Cockerham, 1984) were estimated. A neighbor-joining (NJ) tree was constructed using MEGA 4 (Tamura et al., 2007), and we computed the pairwise standard genetic distance (Ds) (Nei, 1972) using PHYLIP ver. 3.69 (Felsenstein, 2009). Population structure was investigated using the Bayesian clustering algorithm implemented in the STRUCTURE 2.3.3 application (Pritchard et al., 2000). We applied a burn-in period of 20,000 followed by 20,000 iterations of the Markov chain Monte Carlo (MCMC) inference (Pritchard et al., 2000). The probable number of genetic clusters $(K)$ was set to the range of $1 \leq K \leq 15$, with 20 independent runs for each value of $\mathrm{K}$. For the inference of the $\mathrm{K}$ value, we used the estimated likelihood of the probability of data $\operatorname{Ln} \operatorname{Pr}(X / K)$ and the second order rate of change of the likelihood function with respect to $K(\Delta K)$. The $\Delta K$ value was calculated using the Structure Harvester 0.56.3 application (Riztyan et al., 2011). The optimal $K$ value was determined by the greatest $\Delta K$ value as described in Evanno et al. 
Table 2. The genetic variability of nine native chicken populations from Myanmar, Thailand, and Laos

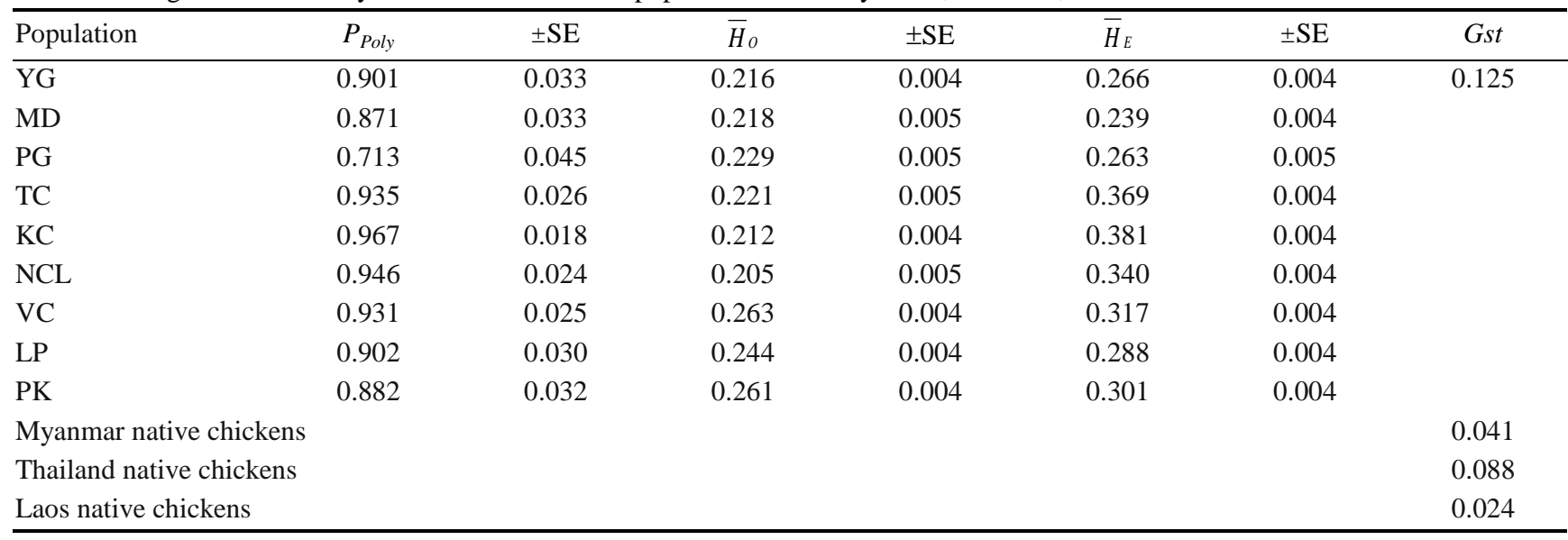

YG, Yangon; MD, Mandalay; PG, Pegu; TC, Trat and Chantaburi, KC, Kanchahaburi; NCL, Nan, Chiang Rai, Lampang; VC, Viangchan; LP, Louangphrabang; PK, Pakxe.

(2005). The DISTRUCT 1.1 application (Rosenberg, 2004) was used to display the probabilities for population subdivisions. Subdivisions were represented as colors, and individuals were depicted as bars divided into color segments corresponding to the membership coefficients for the populations.

\section{RESULTS}

\section{Indels polymorphisms and genetic variability}

The mean ( \pm standard error) Ppoly, $H_{O}$, and $H_{E}$ values for each population are listed in Table 2. The native chickens from the three countries showed polymorphism in most of the indels loci. The highest $(0.967 \pm 0.018)$ and the lowest $(0.713 \pm 0.045)$ Ppoly were found in the KC and PG populations. The average $H_{O}$ ranged from $0.205 \pm 0.005$ in the NCL population to $0.263 \pm 0.004$ in the VC population. The average $H_{E}$ ranged from $0.239 \pm 0.004$ in the $P G$ population to $0.381 \pm 0.004$ in the $\mathrm{KC}$ population. Tests of HWE for all cumulated populations showed HWE at $p<0.05$. In addition, each populations of Myanmar, Thailand, and Laos were in HWE ( $<$ <0.05). The degree of Gst was 0.041 in Myanmar, 0.088 in Thailand, and 0.024 in Laos (Table 2). The Gst among the nine populations was calculated as 0.125 .

\section{Pairwise $\boldsymbol{F}$ st distance and phylogenic analysis}

The pairwise Fst distances are shown in Table 3. The Fst distances between the native chicken populations ranged from 0.045 to 0.094 in Myanmar, from 0.116 to 0.154 in Thai, and from 0.018 to 0.037 in Laos. The pairwise Fst distances between Myanmar and Thailand native chicken populations ranged from 0.194 to 0.308 ; between Myanmar and Laotian, 0.144 to 0.249 ; and between Thai and Laotian, 0.178 to 0.292 . The smallest distance $(0.144)$ was between the YG and PK population, and the largest (0.308) was between the PG and NCL populations.

The NJ tree constructed from the pairwise standard $D s$ between the nine populations is shown in Figure 1. The populations from the three countries were assembled as respective clusters, and the tree is composed of two major clades. The first clade was composed of the Thai and Laotian native chicken populations. The second clade was

Table 3. Pairwise Fst distances among nine native chicken populations from Myanmar, Thailand, and Laos

\begin{tabular}{|c|c|c|c|c|c|c|c|c|c|}
\hline & $\mathrm{YG}$ & MD & $\overline{\mathrm{PG}}$ & $\overline{\mathrm{TC}}$ & $\mathrm{KC}$ & NCL & $\mathrm{VC}$ & $\overline{L P}$ & $\overline{\mathrm{PK}}$ \\
\hline$\overline{Y G}$ & & 0.045 & 0.094 & 0.194 & 0.239 & 0.286 & 0.162 & 0.189 & 0.144 \\
\hline MD & & & 0.073 & 0.202 & 0.244 & 0.291 & 0.168 & 0.196 & 0.165 \\
\hline PG & & & & 0.246 & 0.243 & 0.308 & 0.205 & 0.249 & 0.196 \\
\hline $\mathrm{TC}$ & & & & & 0.127 & 0.154 & 0.178 & 0.218 & 0.201 \\
\hline $\mathrm{KC}$ & & & & & & 0.116 & 0.213 & 0.252 & 0.228 \\
\hline NCL & & & & & & & 0.260 & 0.292 & 0.284 \\
\hline $\mathrm{VC}$ & & & & & & & & 0.028 & 0.018 \\
\hline LP & & & & & & & & & 0.037 \\
\hline PK & & & & & & & & & \\
\hline
\end{tabular}

YG, Yangon; MD, Mandalay; PG, Pegu; TC, Trat and Chantaburi, KC, Kanchahaburi; NCL, Nan, Chiang Rai, Lampang; VC, Viangchan; LP, Louangphrabang; PK, Pakxe. 


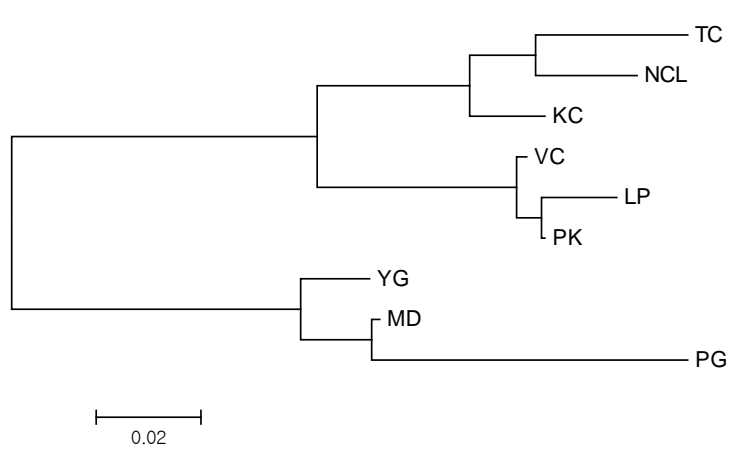

Figure 1. Neighbor-joining (NJ) tree constructed by a genetic distance matrix from nine chicken populations from Myanmar, Thailand, and Laos. YG, Yangon; PG, Pegu; MD, Mandalay; TC, Trat and Chantaburi, KC, Kanchahaburi; NCL, Nan, Chiang Rai Lampang; VC, Viangchan; LP, Louangphrabang; PK, Pakxe.

composed of the Myanmar native chicken populations, which was located outside the first clade.

\section{Population substructure by using structure analysis}

The STRUCTURE analysis revealed that the $\mathrm{Ln} \operatorname{Pr}$ $(X / K)$ at $K=3$ with the maximum likelihood value of $-23,407.74$. The maximum value for the ad hoc statistic, $\Delta K$, was 171.107. Therefore, the probable number of clusters $(K)$ was thought to be three. The contribution of the three ancestral populations $(K=3)$ to the nine populations is presented in Figure 2. The proportion of membership suggested that the native chicken populations from the three countries were descended from three different origins. The presence of admixture was observed among the Thai and Laotian native chicken populations, whereas the Myanmar native chickens lacked admixture.

\section{DISCUSSION}

The results revealed that all of the native chicken populations showed polymorphism in most of the indels loci $(71 \%$ to $96 \%)$. This is larger than that observed in a previous study by Väli et al. (2008). Additionally, their study stated that $81(86.2 \%)$ and $76(80.9 \%)$ out of 94 indels markers could be validated as polymorphic loci in dogs $(\mathrm{n}=7)$ and wolves $(\mathrm{n}=18)$, respectively (Väli et al., 2008). The $H_{E}$ values of chickens native to Myanmar in the present study were similar to those obtained by SNPs in an earlier report by Riztyan et al. (2012). However, for Thai and Laotian native chicken populations, the $H_{E}$ values in the present study were higher than those obtained by SNPs and blood protein polymorphisms in earlier studies (Okamoto et al., 1999; Riztyan et al., 2012). The $H_{E}$ values of Myanmar native chickens were lower than those of the Thai and Laotian populations. Therefore, the genetic variability of the Thai and Laotian native chickens was higher than that of Myanmar populations. This may be linked to the practice of crossbreeding between native chickens and captured Red jungle fowl for cockfighting and as a source of game birds for sport in Thailand and the Philippines (Nishida et al., 2000). Free-scavenging chickens can easily reproduce with wild ones, which may lead to increased genetic variability. There was no HWE deviation among any of the populations or within any populations, suggesting that random mating is predominant among native chickens in those three countries.

The Gst value of Thai native chicken populations was 0.088 , indicating that the degree of genetic differentiation is higher in Thai native chickens than Myanmar and_Laotian native chickens. The Gst values in the present study were higher than those of the chickens native to Myanmar and Laos (0.024 and 0.025), and lower than those of the Thai native chickens (0.127), estimated from the genetic constitution of endogenous protease gene polymorphisms (Okumura et al., 2006). The Gst of Thai native chickens in the present study was also lower than that seen in the study using of 20 microsatellite loci (0.183) of Dorji et al. (2011). The potential source of these variations could be the sample populations, sample sizes, characteristics of different markers and experimental conditions. However, the Gst of the Myanmar and Laotian native chickens was close to the range of 0.001 to 0.039 (Kinoshita et al., 2002), estimated from egg white protein polymorphisms of local populations in Asian countries (Myanmar, Indonesia, China, Nepal, Vietnam, and Laos). The Gst for all the populations was 0.125 , indicating that the genetic differentiation among the native chickens from the three countries was not large. Therefore, Myanmar, Thai, and Laotian native chickens can be regarded as genetically close populations.

The lower pairwise $F$ st distances observed within each country (0.018 to 0.154$)$ and higher pairwise Fst distances

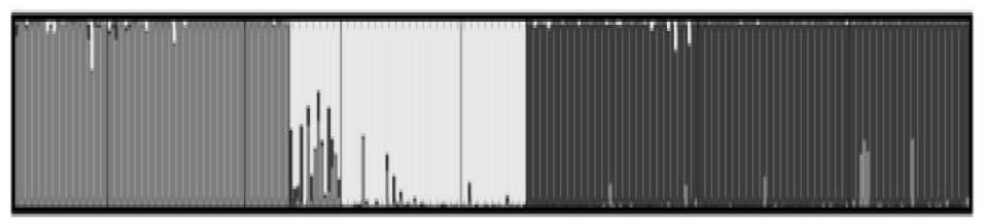

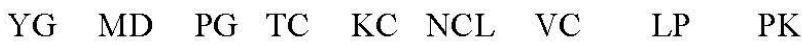

Figure 2. Individual assignment according to the estimated membership coefficient at $K=3$. YG, Yangon; MD, Mandalay; PG, Pegu; TC, Trat and Chantaburi, KC, Kanchahaburi; NCL, Nan, Chiang Rai, Lampang; VC, Viangchan; LP, Louangphrabang; PK, Pakxe. 
observed between countries (0.144 to 0.308$)$, reflect the fact that the populations within the countries were genetically closer populations than those between countries. The pairwise Fst distances between the Thai and Laotian populations were closer than those between the Myanmar and Thai populations. This is consistent with the topology of the NJ tree constructed from $D s$ distances. In the NJ tree, the populations of native chickens from Thailand and Laos were genetically close to one another, while the Myanmar native chickens were genetically distant from them. A similar genetic relationship was oserved by Okumura et al. (2006).

According to the STRUCTURE analysis, native chicken populations from each country were found to have distinct origins because of the long history of domestication in each country. Chickens became domesticated from Red jungle fowl in Southeast Asia nearly 10,000 years ago (Sawai et al., 2010). Interestingly, a small component of admixture from Myanmar populations was found in both Thai and Laotian populations. This was in agreement with the report of Riztyan et al. (2012). In addition, a small component of admixture from Laos was found in Thai populations. However, the admixture from Thai and Laotian populations was not found in Myanmar native chicken populations. The probable causes of admixture from Myanmar to Thai populations may be linked to the influence of Myanmar upon Thailand during the Ayutthaya era, 400 years ago. Myanmar native chickens seem to have been carried along by Myanmar's military (Cocks, 1919; Hmannan Yazawin 2, 1829) and then spread to Laos from Thailand.

The present study examined the genetic diversity and population structure of native chickens from Myanmar, Thailand, and Laos using 102 indels polymorphisms as genetic markers. The native chickens from these three countries demonstrated polymorphisms in most indels loci and are genetically rich diversified populations. The genetic differentiation was higher in Thai native chickens than Myanmar and Laotian populations. According to the NJ tree, Thai and Laotian native chicken populations were genetically closer than Myanmar native chickens. The population structure of the nine chicken populations from the three countries was thought to be based on three different origins $(K=3)$. It was found that genetic admixture was observed in Thai and Laotian native chicken populations. The genetic composition of Myanmar native chickens is pure because of the lack of admixture from the other two countries.

\section{REFERENCES}

Cocks, S. W. 1919. A short history of Burma. 2nd edition. Macmillan and Co. Limited, London, UK.

Dorji, N., M. Daungjinda, and Y. Phasuk. 2011. Genetic characterization of Thai indigenous chickens compared with commercial lines. Trop. Anim. Health Prod. 43:779-785.

Evanno, G., S. Regnaut, and J. Goudet. 2005. Detecting the number of clusters of individuals using the software STRUCTURE: A simulation study. Mol. Ecol. 14:2611-2620.

Felsenstein, J. 2009. Phylogeny Inference Package Version 3.69.

Hmannan Yazawin 2. 1829. Tabinshwehti's Ayutthaya campaign (in Burmese). 2003 edition. Ministry of Information, Yangon, Myanmar.

Kawabe, K., R. Worawut, S. Taura, T. Shimogiri, T. Nishida, and S. Okamoto. 2014. Genetic diversity of mtDNA D-loop polymorphisms in Laotian native fowl populations. Asian Australas. J. Anim. Sci. 27:19-23.

Kinoshita, K., S. Okamoto, T. Shimogiri, K. Kawabe, T. Nishida, R. Kakizawa, Y. Yamamoto, and Y. Maeda. 2002. Gene constitution of egg white proteins of native chicken in Asian countries. Asian Australas. J. Anim. Sci. 15:157-165.

Lewontin, R. C. and J. L. Hubby. 1966. A molecular approach to the study of genetic heterozygosity in natural populations of Drosophila pseduobscura. Genetics 54:595-609.

Liu, Y. P., G. S. Wu, Y. G. Yao, Y. W. Miao, G. Luikart, M. Baig, A. Beja-Pereira, Z. L. Ding, M. G. Palanichamy, and Y. P. Zhang. 2006. Multiple maternal origins of chickens: Out of the Asian jungles. Mol. Phylogenet. Evol. 38:12-19.

Maw, A. A., T. Shimogiri, Riztyan, K. Kawabe, Y. Kawamoto, and S. Okamoto. 2012. Genetic diversity of Myanmar and Indonesia native chickens together with two jungle fowl species by using 102 Indels polymorphisms. Asian Australas. J. Anim. Sci. 25:927-934.

Mwacharo, J. M., H. Jianlin, and T. Amano. 2006. Native African chicken: valuable genetic resources for future breeding improvement. J. Anim. Genet. Jpn. 34:63-69.

Nishibori, M., T. Shimogiri, T. Hayashi, and H. Yasue. 2005. Molecular evidence of hybridization of species in the genus Gallus except of Gallus varius. Anim. Genet. 36:367-375.

Natalle, S. C. F, R. L. Resque, E. M. Ribeiro-Rodrigues, J. F. Guerreiro, N. P. C. Santos, A. R. Santos, and S. Santos. 2010. $\mathrm{X}$-linked insertion/deletion polymorphisms: Forensic applications of a 33-markers panel. Int. J. Legal Med. 124: 589-593.

Nei, M. 1973. Analysis of gene diversity in subdivided populations. Proc. Natl. Acad. Sci. USA. 70:3321-3323.

Nei, M. 1978. Estimation of average heterozygosity and genetic distance from a small number of individuals. Genetics 89:583590.

Nishida, T., W. Rerkamnuaychoke, D. G. Tung, S. Saignaleus, S. Okamoto, Y. Kawamoto, J. Kimura, K. Kawabe, N. Tsunekawa, H. Otaka, and Y. Hatashi. 2000. Morphological identification and ecology of Red Jungle Fowl in Thailand, Laos and Vietnam. Anim. Sci. J. 71:470-480.

Okamoto, S., N. Tsunekawa, Y. Kawamoto, R. Worawut, K. Kawabe, Y. Maeda, and T. Nishida. 1999. Blood protein polymorphisms of native fowls in Laos. Asian Australas. J. Anim. Sci. 12:1011-1014.

Okamoto, S., K. Kinoshita, Y. Yamamoto, K. Tsunoda, H. Okabayashi, H. Mannen, K. Tanaka, T. Yamagata, K. Nozawa, M. Nishibori, Y. Kurosawa, Maung Maung Nyunt, Than Daing, Than Hla, Nay Win, and Y. Maeda. 2004. Blood protein variations of native fowls in Myanmar. Researches Report of the Society for Native Livestock. 21:237-242. 
Okumura, F., T. Shimogiri, K. Kawabe, S. Okamoto, M. Nishibori, Y. Yamamoto, and Y. Maeda. 2006. Gene constitution of South-East Asian native Chickens, commercial chickens and jungle fowl using polymorphisms of four Calpain genes. Anim. Sci. J. 77:188-195.

Pritchard, J. K., M. Stephens, and P. Donnelly. 2000. Inference of population structure using multilocus genotype data. Genetics 155:945-959.

Riztyan, T. Katano, T. Shimogiri, K. Kawabe, and S. Okamoto. 2011. Genetic diversity and population structure of Indonesian native chickens based on single nucleotide polymorphism makers. Poult. Sci. 90: 2471-2478.

Riztyan, T. Nishida, R. Worawut, T. Shimogiri, A. A. Maw, K. Kawabe, Y. Kawamoto, and S. Okamoto. 2012. Genetic variation and phylogeographic analysis of native chicken populations in Myanmar and Thailand. J. Poult. Sci. 49:68-73.

Rosenberg, N. A. 2004. DISTRUCT: a program for the graphical display of population structure. Mol. Ecol. Notes 4:137-138.
Sawai, H., H. L. Kim, K. Kuno, S. Suzuki, H. Gotoh, M. Takada, N. Takahata, Y. Satta, and F. Akishinonomiya. 2010. The origin and genetic variation of domestic chickens with special reference to jungle fowls Gallus g. gallus and G. varius. PLoS One. 5:e10639.

Sambrook, J., E. F. Fritsch, and T. Maniatis. 1989. Molecular cloning: A laboratory manual, 2nd edition, Cold Spring Laboratory Press, Cold Spring Harbor, New York, USA.

Tamura, K., J. Dudley, M. Nei, and S. Kumar. 2007. MEGA4: Molecular evolutionary genetics analysis (MEGA) software version 4.0. Mol. Biol. Evol. 24:1596-1599.

Väli, U., M. Brandström, M. Johansson, and H. Ellegren. 2008. Insertion-deletion polymorphisms (indels) as genetic markers in natural populations. BMC Genet. 9:8.

Weir, B. S. 1996. Genetic Data Analysis II. Sinauer. Sunder land, MA, USA.

Weir, B. S. and C. C. Cockerham. 1984. Estimating F-statistic for the analysis of population structure. Evolution 38:1358-1370. 